\title{
Effect of Plant Extract Combinations on Some Bacterial Pathogens
}

\author{
${ }^{*}$ OBUEKWE, IS; ${ }^{1}$ OKOYOMO, EP; ${ }^{2}$ ANKA, US
}

\author{
${ }^{*}$ Department of Microbiology, Faculty of Life Sciences, University of Benin, Benin City, Edo State, Nigeria \\ ${ }^{2}$ Department of Geography, Faculty of Earth and Environmental Sciences, Bayero University, Kano State, Nigeria \\ *Corresponding Author Email: ifeyinwa.obuekwe@uniben.edu
}

\begin{abstract}
Increase in antimicrobial resistance coupled with successful treatment of various diseases with herbal medications has triggered the upsurge in research geared towards harnessing the medicinal potentials of various plants. The aim of this study was to evaluate the phytochemical composition of Bryophyllum pinnatum, Ocimum gratissimum, Jatropha curcas and Ficus exasperata and their combined antibacterial activity on Escherichia coli, Salmonella typhi, Staphylococcus aureus, and Klebsiella pneumonia. Plant leaves were air-dried, extracted with ethyl-acetate and water with subsequent qualitative analyses for phytochemical compounds. Bacterial pathogens were obtained from University of Benin Teaching Hospital (UBTH), confirmed, and their zones of inhibition studied at 50, 40, 30, 20 and $10 \mathrm{mg} / \mathrm{ml}$ of extract combinations. The presence of phenols, tannins and saponins were confirmed in all extracts while alkaloids were present in all ethyl acetate extracts and terpenoids in all aqueous extracts. Different zones of inhibition were measured with the different extract combinations with $E$. coli having its highest zone of inhibition with the combination of Bryophyllum pinnatum and Ocimum gratissimum in $30 \mathrm{mg} / \mathrm{ml}(27.25 \pm 1.70 \mathrm{~mm})$. However, the highest zone of inhibition observed in the study was with S. aureus in $10 \mathrm{mg} / \mathrm{ml}$ concentration of ethyl acetate plant extracts of Ocimum gratissimum and Ficus exasperata $(31.75 \pm 3.07 \mathrm{~mm})$. The Gram positive cell wall of $S$. aureus is less complex and therefore, more susceptible to bio-agents. Antibacterial activities of these extracts are attributed to the presence of secondary metabolites that make them good bio-agents for production of antibacterial drugs.
\end{abstract}

DOI: https://dx.doi.org/10.4314/jasem.v24i4.13

Copyright: Copyright (C) 2020 Obuekwe et al. This is an open access article distributed under the Creative Commons Attribution License (CCL), which permits unrestricted use, distribution, and reproduction in any medium, provided the original work is properly cited.

Dates: Received: 11 February 2020; Revised: 14 March 2020; Accepted: 22 March 2020

Keywords: Plant extracts, Inhibition zones, Escherichia coli, Staphylococcus aureus

Pharmacological industries have been left with the challenge of occasional emergence of bacterial strains with the ability to overcome the effect of antibiotics previously used to treat them (Odey et al., 2012; Awala et al., 2017). Resistance to these drugs by bacteria has resulted in the need to source for alternative antibacterial agents such as plant extracts (WHO, 2000; Costa et al., 2015; Ildiz et al., 2018). Plants of medicinal importance contain huge varieties of phytochemicals which possess important therapeutic properties that can be used for the treatment of emerging and re-emerging human diseases (Abbas et al., 2017; Tyagi et al., 2017; Kin et al., 2018). Phytochemical substances such as alkaloids, essential oils, peptides, tannins, phenols and flavonoids are medicinal agents responsible for the antimicrobial ability of the plants especially against disease causing microorganisms (Odey et al., 2012; Costa et al., 2015; Udochukwu et al., 2015; Rukmini et al., 2017; Aggarwal et al., 2017).

Leaf extracts of Occimium gratissimum (scent leaf), Fiscus exasperata Vahl (sand paper tree), Jathropha curcas (purging nut) and Bryophyllum pinnatum (miracle plant) have been singly analyzed as natural antimicrobials against various pathogenic organisms such as Staphylococcus aureus, Escherichia coli, Streptococcus fecalis and Pseudomonas aeruginosa (Terezinha et al., 2008; Akinjogunla et al., 2009; Ladipo et al., 2010. The efficacy of these medicinal plants however, depends on their solubility in solvents of different polarity (Odey et al., 2012; Zlotek et al., 2016). Plants antimicrobials have been found to be synergistic enhancers in that though they may have small antimicrobial properties alone, when taken concurrently with other plants enhance these effects (Rakholiya and Chanda, 2012). In this regard, this study aimed at analyzing the phytochemical extracts of Occimium gratissimum, Ficus exasperata, Jathropha curcas and Bryophyllum pinnatum and their combined antibacterial activities against selected human bacterial pathogens.

\section{MATERIALS AND METHODS}

Collection of Plant Materials: The fresh leaves of Bryophyllum pinnatum, Ocimum gratissimum, Jatropha curcas and Ficus exasperata were collected from Sapele road, Benin City, Nigeria. The plants 
were identified and authenticated by the Department of Plant Biology and Biotechnology, Faculty of Life Sciences, University of Benin, Benin City.

Preparation of plant materials: The fresh leaves were rinsed and shade dried to a constant weight over a period of time. The dried leaves were pulverized using British mechanical grinder. The powdered leaves weighed $150 \mathrm{~g}$ per plant and were soaked separately in $500 \mathrm{ml}$ of ethyl-acetate and aqua for 72 hours using cold method of maceration. The macerated materials were filtered, and the filtrate concentrated to dryness under reduced temperature in water bath using crucibles. The dried extracts were stored in a separate air-tight clean glass container at $4{ }^{\circ} \mathrm{C}$ for further use.

Collection of bacterial pathogens: Staphylococcus aureus, Escherichia coli, Salmonella typhi and Klebsiella pneumonia were obtained from University of Benin Teaching Hospital (UBTH) and were put in slants and stored in a refrigerator until use. The isolates were confirmed in the Department of Microbiology, University of Benin

Qualitative phytochemical analyses of plants extracts: Standard procedures were used to detect and quantify flavonoids, alkaloids, phlobatannins, phenolic compounds, tannins, saponins, cardiac glycosides, and terpenoids compounds in the four plants extracts (Harborne, 1973; Obadoni and Ochuko, 2001)

Preparation of Extract Combinations: The four plant leaves of Bryophyllum pinnatum (B), Ocimum gratissimum (O), Jatropha curcas (J) and Ficus exasperata (F) extracts of ethyl-acetate and aqua made up to eight extracts which were combined separately in twos, threes and a four in five concentrations of 50 , 40, 30, 20 and $10 \mathrm{mg} / \mathrm{ml}$. These combinations in twos were BO, BJ, BF, OJ, OF, JF; in threes they were BOJ, $\mathrm{BOF}, \mathrm{BJF}, \mathrm{OJF}$ and in four was BOJF.

Antibacterial Activity of different combined Extracts: The mixtures of the different dissolved plant extracts combinations were stirred with a sterile glass rod and left to stand for 20 minutes. Discs (sterilized Whatmann no. 1 filter paper cut with a hand paperpunch) were introduced into the mixture, and were left to soak for 2 hours before introducing them in plates containing the bacterial pathogens.

Antibacterial Assay: Pathogenic bacterial isolates growing on nutrient agar plates were picked to make suspensions in $1 \mathrm{ml}$ sterile normal saline and this was adjusted to an equivalence of a 0.5 McFarland standard. Sterile Mueller-Hinton agar (Oxoid, UK) plates were inoculated by spreading $0.1 \mathrm{ml}$ of each bacteria inoculum suspension on the entire surface of the plate and the extracts impregnated discs were inserted. Inoculated plates with discs impregnated with crude extracts were placed in an incubator at 37 ${ }^{\circ} \mathrm{C}$ for 24 hours. The zones of inhibition were measured using a ruler to the nearest millimetre and recorded.

Statistical Analysis: Zones of inhibition ( $\mathrm{mm}$ ) of the different plants extracts combinations were done in replicates and means with standard errors were calculated using the Statistical Package for Social Sciences software (SPSS version 20). Hypothesis testing using t-Test was conducted at $\mathrm{p}<0.05$ confidence level (Ogbeibu, 2014).

\section{RESULTS AND DISCUSSION}

Four different plant leaves of Bryophyllum pinnatum, Ocimum gratissimum, Jatropha curcas and Ficus exasperata were extracted with both Ethyl acetate and aqua, and their phytochemical contents analyzed. Both extractants pooled out tannins, saponins and phenols from all plants leaves (Tables 1 and 2). This was not surprising because plant extracts contain metabolites such as phenolics, terpenes and alkaloids that show both biological and pharmacological activities (Stefanovic and Comic, 2012; Kin et al., 2018). However, alkaloids were only present in plant leaves extracted with Ethyl acetate and in Ficus exasperata in the aqueous extract. Similarly, Awala et al. (2017) showed the presence of phenols, tannins and alkaloids in acetone and methanol extracted leaves of Ficus exasperata. Conversely, aqueous extracts of all plants leaves contained terpenoids but this was only observed in B. pinnatum extracted in Ethyl acetate (Tables 1 and 2). Kin et al. (2018) similarly reported the absence of terpenoids in methanol extracted $O$. gratissimum. Bryophyllum pinnatum (B), Ocimum gratissimum $(\mathrm{O})$, Jatropha curcas (J) and Ficus exasperata (F) Ethyl acetate and aqua leaves extracts at 50, 40, 30, 20 and $10 \mathrm{mg} / \mathrm{ml}$ concentrations were combined in twos, threes, and a four and their antibacterial activities were assessed (Tables 3 and 4). Zones of inhibition on bacterial lawn incorporated with plant extracts discs were used to measure antibacterial activities. For $E$. coli, the highest zones of inhibition measured where in the two combinations of Bryophyllum pinnatum and Ocimum gratissimum in $30 \mathrm{mg} / \mathrm{ml}(27.25 \pm 1.70 \mathrm{~mm})$ and the four combinations of Bryophyllum pinnatum, Ocimum gratissimum, Jatropha curcas and Ficus exasperata $(26.50 \pm 1.55)$ in $10 \mathrm{mg} / \mathrm{ml}$ using Ethyl acetate (Table 3). Similarly, aqua extracts of Bryophyllum pinnatum, Ocimum gratissimum and Jatropha curcas in $30 \mathrm{mg} / \mathrm{ml}(20.00 \pm 1.78)$ had the highest zone of inhibition. 
Table 1: Qualitative phytochemical constituent of Ethyl acetate extracts across various plants

\begin{tabular}{lcccc}
\hline & \multicolumn{5}{c}{ Plant Extracts } \\
\cline { 2 - 6 } Phytochemicals & B. pinnatum & O. gratissimum & J. curcas & F. exerspifolia \\
\hline Alkaloids & + & + & + & + \\
Flavonoids & + & + & + & + \\
Tannins & + & + & + & + \\
Phlobatannins & - & - & - & - \\
Saponins & + & + & + & + \\
Terpenoids & + & - & - & - \\
Cardiac glycosides & + & + & + & + \\
Phenols & + & + & + & + \\
\hline \multicolumn{5}{c}{} \\
\cline { 2 - 6 } & Keys: & + indicates present & - indicates absent
\end{tabular}

Table 2: Qualitative phytochemical constituent of Aqueous extracts across various plants

\begin{tabular}{|c|c|c|c|c|}
\hline \multirow{2}{*}{ Phytochemicals } & \multicolumn{4}{|c|}{ Plant Extracts } \\
\hline & B. pinnatum & O. grattissimum & J. curcas & F. exasperata \\
\hline Alkaloids & - & - & - & + \\
\hline Flavonoids & + & - & + & + \\
\hline Tannins & + & + & + & + \\
\hline Phlobatannins & - & + & + & - \\
\hline Saponins & + & + & + & + \\
\hline Terpenoids & + & + & + & + \\
\hline Cardiac glycosides & - & + & - & + \\
\hline Phenols & + & + & + & + \\
\hline
\end{tabular}

These observed zones of inhibition are as a result of secondary metabolites in the leave extracts such as alkaloids, flavonoids, phenols and essential oils which affect bacterial sensitivity to medicinal plants (Dawoud et al., 2013; Ildiz et al., 2018; Kin et al., 2018). Higher zones of bacterial inhibition was observed in this study as compared to that of E. coli, $P$. aeruginosa and $S$. aureus at $100 \mu 1 / \mathrm{ml}(7.0-11.5$ $\mathrm{mm})$ reported with ethanol and aqueous extracts of $O$. gratissimum (Udochukwu et al., 2015). This is likely as a result of combined effects of the plant extracts in relation to only a single extract. Plants extracts may interact with one another to improve their solubility, and thereby enhance their bioavailability and subsequent antibacterial activity (Chouhan et al., 2017). However, combinations of BJF and OJ at lower concentrations using Acetyl acetate were not very effective in inhibiting E.coli. This could be as a result of lower concentrations of the phytochemicals at these extract combinations. The highest zone of inhibition observed in $S$. enterica when Ethyl acetate plants extracts were introduced was with BOF $(27.00 \pm 1.23$ $\mathrm{mm}$ ) combinations in $20 \mathrm{mg} / \mathrm{ml}$ concentration while this was in $30 \mathrm{mg} / \mathrm{ml}$ in BOJ $(30.00 \pm 3.54)$ combination using aqueous extract (Table 4). Three combinations of the plant extracts had better antibacterial activity against $S$. enterica as compared to the twos and the four combinations. Ildiz et al. (2018) showed that the combination of cefoxitin with coriander extracts had stronger effect than cefoxitin alone on Methicillin Resistant Staphylococcus aureus (MRSA), E.coli and K. pneumonia. The different combinations of plant extracts showed varied zones of antibacterial activity against $K$. pneumonia. However, the highest zones of inhibition were observed in 20 $\mathrm{mg} / \mathrm{ml}$ concentration of both Acetyl acetate and aqueous extracts. Although this was with three combinations of BOF $(20.00 \pm 3.54)$ in the former and in two combinations of $\mathrm{BF}(26.25 \pm 2.39)$ in the later. This is similar to the work of Ildiz et al. (2018) where $25 \mathrm{~mm}$ zone of inhibition of $K$. pneumoniae with petroleum ether extracted coriander seed was reported. Interestingly, the highest antibacterial activity observed in the study was against $S$. aureus and this was observed in $10 \mathrm{mg} / \mathrm{ml}$ concentration of Ethyl acetate plant extracts of $\mathrm{OF}(31.75 \pm 3.07)$ and in 50 $\mathrm{mg} / \mathrm{ml}$ of BOJF $(30.00 \pm 0.00)$ aqueous extract. This agrees with the findings of Awala et al. (2017) who reported $31.27 \mathrm{~mm}$ zone of inhibition of Ficus exasperata acetone and methanol extract against $S$. aureus. The high antibacterial activity against $S$. aureus could be because of the Gram positive cell wall which is less complex and more susceptible to bioagents as compared to that of the Gram negative bacteria (E. coli, S. typhi, $S$. and $K$. pneumonia). Several authors have reported that plant extracts can act synergistically against bacterial pathogens of public health importance (Rojas et al., 2004; Prakash et al., 2006; Karmegam et al., 2008; D'Arrigo et al., 2010). Combined effect of ethanolic leaf extracts of Catharanthus roseus, Lawsonia inermis and Chrysanthemum odoratum exerted a higher activity against MRSA as compared to single leaf extracts. On the whole, the leave extracts in this study are versatile in bioactive agents and demonstrate antibacterial activities against the studied human pathogens. 
Table 3: Synergistic effect of Ethyl acetate extracts of plants against selected bacterial pathogens

\begin{tabular}{|c|c|c|c|c|c|c|c|c|c|c|c|c|}
\hline \multirow[t]{2}{*}{ Pathogens } & \multirow{2}{*}{$\begin{array}{l}\text { Conc. } \\
(\mathrm{mg} / \mathrm{ml})\end{array}$} & \multicolumn{11}{|c|}{ Zone of Inhibition (mm) of Extract Combinations } \\
\hline & & $\mathrm{BO}$ & $\mathrm{BJ}$ & $\mathrm{BF}$ & $\mathrm{BOJ}$ & $\mathrm{BOF}$ & $\mathrm{BJF}$ & $\mathrm{BOJF}$ & $\mathrm{OJ}$ & $\mathrm{OF}$ & OJF & $\mathrm{JF}$ \\
\hline \multirow{5}{*}{ E. coli } & 50 & $10.25 \pm 0.25^{\mathrm{b}}$ & $13.25 \pm 0.63^{\mathrm{e}}$ & $10.75 \pm 0.48^{\mathrm{b}}$ & $12.50 \pm 0.65^{\mathrm{d}}$ & $12.00 \pm 0.41^{\mathrm{c}}$ & $8.75 \pm 0.48^{\mathrm{a}}$ & $10.25 \pm 0.25^{\mathrm{b}}$ & $11.25 \pm 0.63^{\mathrm{b}}$ & $10.00 \pm 0.00^{\mathrm{a}}$ & $9.75 \pm 0.25^{\mathrm{a}}$ & $11.25 \pm 0.63^{\mathrm{b}}$ \\
\hline & 40 & $15.25 \pm 0.63^{\mathrm{d}}$ & $11.00 \pm 0.00^{\mathrm{c}}$ & $10.50 \pm 0.29^{\mathrm{b}}$ & $10.50 \pm 0.50^{\mathrm{b}}$ & $10.25 \pm 0.25^{\mathrm{b}}$ & $0.00 \pm 0.00^{\mathrm{a}}$ & $9.50 \pm 0.29^{\mathrm{b}}$ & $18.25 \pm 0.63^{\mathrm{e}}$ & $9.50 \pm 0.29^{\mathrm{b}}$ & $15.75 \pm 0.63^{\mathrm{d}}$ & $11.00 \pm 0.29^{\circ}$ \\
\hline & 30 & $27.25 \pm 1.70^{\mathrm{f}}$ & $10.75 \pm 0.48^{\mathrm{b}}$ & $10.00 \pm 0.41^{\mathrm{b}}$ & $11.25 \pm 0.95^{\mathrm{b}}$ & $17.00 \pm 1.23^{\mathrm{e}}$ & $0.00 \pm 0.00^{\mathrm{a}}$ & $10.00 \pm 0.00^{\mathrm{b}}$ & $0.00 \pm 0.00^{\mathrm{a}}$ & $12.75 \pm 0.48^{\mathrm{c}}$ & $13.50 \pm 1.04^{\mathrm{d}}$ & $10.75 \pm 0.48^{\mathrm{b}}$ \\
\hline & 20 & $14.00 \pm 0.82^{\mathrm{d}}$ & $9.50 \pm 0.29^{\mathrm{b}}$ & $9.75 \pm 0.48^{\mathrm{b}}$ & $8.25 \pm 2.43^{\mathrm{b}}$ & $10.00 \pm 0.00^{\mathrm{b}}$ & $0.00 \pm 0.00^{\mathrm{a}}$ & $10.75 \pm 0.48^{\mathrm{b}}$ & $0.00 \pm 0.00^{\mathrm{a}}$ & $8.50 \pm 0.85^{\mathrm{b}}$ & $12.75 \pm 0.25^{\mathrm{c}}$ & $9.75 \pm 0.25^{\mathrm{b}}$ \\
\hline & 10 & $9.50 \pm 0.29^{\mathrm{b}}$ & $8.50 \pm 0.29^{\mathrm{b}}$ & $8.25 \pm 0.25^{\mathrm{b}}$ & $13.50 \pm 0.96^{\mathrm{d}}$ & $13.00 \pm 0.58^{\mathrm{d}}$ & $0.00 \pm 0.00^{\mathrm{a}}$ & $26.50 \pm 1.55^{\mathrm{e}}$ & $0.00 \pm 0.00^{\mathrm{a}}$ & $9.50 \pm 0.29^{\mathrm{b}}$ & $11.00 \pm 0.41^{\mathrm{c}}$ & $10.75 \pm 0.25^{\circ}$ \\
\hline \multirow{5}{*}{ S. enterica } & 50 & $11.75 \pm 0.48^{\mathrm{a}}$ & $11.25 \pm 0.75^{\mathrm{a}}$ & $9.50 \pm 0.65^{\mathrm{a}}$ & $12.75 \pm 0.75^{\mathrm{b}}$ & $18.75 \pm 1.44^{\mathrm{c}}$ & $12.75 \pm 0.25^{\mathrm{b}}$ & $12.75 \pm 0.48^{\mathrm{b}}$ & $12.50 \pm 0.50^{\mathrm{b}}$ & $17.00 \pm 1.23^{\mathrm{c}}$ & $10.00 \pm 0.41^{\mathrm{a}}$ & $10.75 \pm 0.48^{a}$ \\
\hline & 40 & $14.00 \pm 0.41^{\mathrm{d}}$ & $13.75 \pm 0.75^{\mathrm{c}}$ & $10.75 \pm 0.75^{\mathrm{a}}$ & $12.75 \pm 0.25^{\mathrm{b}}$ & $18.75 \pm 1.89^{\mathrm{e}}$ & $10.75 \pm 0.48^{\mathrm{a}}$ & $9.50 \pm 0.29^{\mathrm{a}}$ & $11.25 \pm 0.25^{\mathrm{a}}$ & $12.25 \pm 1.11^{\mathrm{b}}$ & $10.25 \pm 0.63^{\mathrm{a}}$ & $9.25 \pm 0.48^{\mathrm{a}}$ \\
\hline & 30 & $17.50 \pm 1.19^{\mathrm{g}}$ & $11.50 \pm 1.04^{b}$ & $14.50 \pm 0.50^{\mathrm{e}}$ & $17.25 \pm 1.60^{\mathrm{g}}$ & $12.25 \pm 0.25^{\mathrm{c}}$ & $0.00 \pm 0.00^{\mathrm{a}}$ & $10.25 \pm 0.25^{\mathrm{b}}$ & $16.50 \pm 0.96^{\mathrm{f}}$ & $10.25 \pm 0.25^{\mathrm{b}}$ & $14.00 \pm 1.00^{\mathrm{d}}$ & $9.25 \pm 0.48^{\mathrm{b}}$ \\
\hline & 20 & $15.25 \pm 2.06^{\mathrm{d}}$ & $9.75 \pm 0.25^{\mathrm{a}}$ & $10.00 \pm 0.00^{\mathrm{a}}$ & $11.25 \pm 0.48^{\mathrm{a}}$ & $27.00 \pm 1.23^{\mathrm{e}}$ & $8.75 \pm 0.25^{\mathrm{a}}$ & $11.50 \pm 0.29^{\mathrm{b}}$ & $13.00 \pm 0.82^{\mathrm{c}}$ & $9.25 \pm 0.25^{\mathrm{a}}$ & $11.00 \pm 0.71^{\mathrm{a}}$ & $10.25 \pm 0.25^{a}$ \\
\hline & 10 & $9.25 \pm 0.25^{\mathrm{b}}$ & $4.00 \pm 2.31^{\mathrm{a}}$ & $9.75 \pm 0.25^{\mathrm{b}}$ & $11.50 \pm 0.87^{\mathrm{b}}$ & $16.75 \pm 1.18^{\mathrm{c}}$ & $9.25 \pm 0.48^{\mathrm{b}}$ & $15.00 \pm 0.00^{\mathrm{c}}$ & $10.25 \pm 0.25^{\mathrm{b}}$ & $9.75 \pm 0.25^{\mathrm{b}}$ & $10.50 \pm 0.65^{\mathrm{b}}$ & $10.75 \pm 0.48^{b}$ \\
\hline \multirow{5}{*}{$\begin{array}{l}\text { K } \\
\text { pneumonia } \\
e\end{array}$} & 50 & $10.00 \pm 0.00^{\mathrm{a}}$ & $10.25 \pm 0.25^{\mathrm{a}}$ & $9.75 \pm 0.25^{\mathrm{a}}$ & $10.00 \pm 0.00^{\mathrm{a}}$ & $14.75 \pm 0.25^{\mathrm{d}}$ & $10.75 \pm 0.48^{\mathrm{a}}$ & $11.25 \pm 0.48^{\mathrm{b}}$ & $10.00 \pm 0.41^{\mathrm{a}}$ & $10.75 \pm 0.25^{\mathrm{a}}$ & $12.25 \pm 0.95^{\mathrm{c}}$ & $12.25 \pm 0.63^{\mathrm{c}}$ \\
\hline & 40 & $9.25 \pm 0.25^{\mathrm{a}}$ & $9.75 \pm 0.25^{\mathrm{b}}$ & $9.50 \pm 0.48^{\mathrm{b}}$ & $11.25 \pm 0.41^{\mathrm{d}}$ & $11.50 \pm 0.48^{\mathrm{d}}$ & $8.25 \pm 0.48^{\mathrm{a}}$ & $9.25 \pm 0.41^{\mathrm{a}}$ & $10.00 \pm 0.48^{\mathrm{b}}$ & $10.00 \pm 0.00^{\mathrm{b}}$ & $10.75 \pm 0.48^{\mathrm{c}}$ & $10.00 \pm 0.41^{\mathrm{b}}$ \\
\hline & 30 & $8.75 \pm 0.48^{\mathrm{a}}$ & $7.75 \pm 0.28^{\mathrm{a}}$ & $16.75 \pm 1.03^{\mathrm{f}}$ & $14.00 \pm 0.00^{\mathrm{e}}$ & $12.75 \pm 0.25^{\mathrm{d}}$ & $9.25 \pm 0.25^{\mathrm{a}}$ & $8.50 \pm 0.28^{\mathrm{a}}$ & $11.50 \pm 1.19^{\mathrm{c}}$ & $8.50 \pm 0.29^{\mathrm{a}}$ & $10.00 \pm 0.41^{\mathrm{b}}$ & $10.00 \pm 0.00^{\mathrm{b}}$ \\
\hline & 20 & $7.50 \pm 0.29^{\mathrm{b}}$ & $8.00 \pm 0.41^{\mathrm{b}}$ & $9.25 \pm 0.25^{\mathrm{b}}$ & $12.00 \pm 0.41^{\mathrm{c}}$ & $20.00 \pm 3.54^{\mathrm{d}}$ & $0.00 \pm 0.00^{\mathrm{a}}$ & $8.50 \pm 0.29^{\mathrm{a}}$ & $10.00 \pm 0.41^{\mathrm{b}}$ & $8.00 \pm 0.41^{\mathrm{b}}$ & $10.75 \pm 0.48^{\mathrm{b}}$ & $9.50 \pm 0.29^{b}$ \\
\hline & 10 & $8.75 \pm 0.25^{\mathrm{b}}$ & $7.75 \pm 0.25^{\mathrm{b}}$ & $7.50 \pm 0.29^{\mathrm{b}}$ & $13.25 \pm 1.03^{\mathrm{e}}$ & $12.25 \pm 0.95^{\mathrm{d}}$ & $0.00 \pm 0.00^{\mathrm{a}}$ & $8.25 \pm 0.48^{\mathrm{b}}$ & $16.25 \pm 1.32^{\mathrm{f}}$ & $7.75 \pm 0.25^{\mathrm{b}}$ & $10.75 \pm 0.75^{\mathrm{c}}$ & $9.25 \pm 0.25^{\mathrm{b}}$ \\
\hline \multirow{5}{*}{ S. aureus } & 50 & $12.25 \pm 0.95^{\mathrm{e}}$ & $9.00 \pm 0.41^{\mathrm{a}}$ & $10.00 \pm 0.00^{\mathrm{a}}$ & $12.00 \pm 0.00^{\mathrm{d}}$ & $12.50 \pm 0.87^{\mathrm{e}}$ & $11.25 \pm 0.48^{\mathrm{c}}$ & $13.25 \pm 0.63^{\mathrm{f}}$ & $10.75 \pm 0.75^{b}$ & $8.50 \pm 0.65^{\mathrm{a}}$ & $11.00 \pm 0.71^{\mathrm{c}}$ & $10.25 \pm 0.25^{a}$ \\
\hline & 40 & $21.50 \pm 0.96^{\mathrm{d}}$ & $10.00 \pm 0.41^{\mathrm{a}}$ & $11.00 \pm 0.41^{\mathrm{a}}$ & $11.50 \pm 0.29^{\mathrm{a}}$ & $13.50 \pm 1.19^{\mathrm{b}}$ & $11.75 \pm 0.63^{\mathrm{a}}$ & $16.75 \pm 1.32^{\mathrm{c}}$ & $10.25 \pm 0.25^{\mathrm{a}}$ & $10.00 \pm 0.00^{\mathrm{a}}$ & $16.00 \pm 1.41^{\mathrm{c}}$ & $10.25 \pm 0.48^{a}$ \\
\hline & 30 & $23.00 \pm 1.73^{\mathrm{d}}$ & $9.75 \pm 0.25^{\mathrm{a}}$ & $9.00 \pm 0.00^{\mathrm{a}}$ & $14.25 \pm 0.25^{\mathrm{b}}$ & $14.50 \pm 0.29^{\mathrm{b}}$ & $9.75 \pm 0.25^{\mathrm{a}}$ & $18.00 \pm 0.71^{\mathrm{c}}$ & $11.25 \pm 0.95^{\mathrm{a}}$ & $18.75 \pm 0.75^{\mathrm{c}}$ & $9.75 \pm 0.25^{\mathrm{a}}$ & $9.75 \pm 0.25^{\mathrm{a}}$ \\
\hline & 20 & $22.50 \pm 1.50^{\mathrm{c}}$ & $9.00 \pm 0.00^{\mathrm{a}}$ & $11.25 \pm 0.25^{\mathrm{a}}$ & $10.75 \pm 0.25^{\mathrm{a}}$ & $19.75 \pm 1.93^{b}$ & $10.00 \pm 0.41^{\mathrm{a}}$ & $25.00 \pm 0.00^{\mathrm{d}}$ & $11.50 \pm 0.29^{\mathrm{a}}$ & $10.25 \pm 0.25^{\mathrm{a}}$ & $9.25 \pm 0.25^{\mathrm{a}}$ & $9.25 \pm 0.25^{\mathrm{a}}$ \\
\hline & 10 & $14.00 \pm 0.71^{\mathrm{c}}$ & $8.50 \pm 0.29^{\mathrm{a}}$ & $10.00 \pm 0.41^{\mathrm{a}}$ & $10.00 \pm 0.00^{\mathrm{a}}$ & $10.50 \pm 0.29^{\mathrm{a}}$ & $12.00 \pm 0.58^{\mathrm{a}}$ & $13.25 \pm 0.63^{\mathrm{b}}$ & $22.00 \pm 1.78^{\mathrm{d}}$ & $31.75 \pm 3.07^{\mathrm{e}}$ & $11.25 \pm 0.63^{\mathrm{a}}$ & $8.25 \pm 0.25^{\mathrm{a}}$ \\
\hline
\end{tabular}

Key: B: Bryophyllum pinnatum; O: Ocimum gratissimum; J: Jathropha curcas; F: Ficus experata; a-f: different characters in the same row indicate values with significant difference ( $<<0.05$ )

Table 4: Synergistic effect of aqueous extracts of plants against selected bacterial pathogens

\begin{tabular}{|c|c|c|c|c|c|c|c|c|c|c|c|c|}
\hline \multirow[t]{2}{*}{ Pathogens } & \multirow{2}{*}{$\begin{array}{l}\text { Conc. } \\
\text { (mg/ml) }\end{array}$} & \multicolumn{11}{|c|}{ Zone of Inhibition (mm) of Extract Combinations } \\
\hline & & $\mathrm{BO}$ & BJ & $\mathrm{BF}$ & BOJ & $\mathrm{BOF}$ & BJF & BOJF & OJ & $\mathrm{OF}$ & OJF & $\mathrm{JF}$ \\
\hline \multirow{5}{*}{ E. coli } & 50 & $10.25 \pm 0.48^{\mathrm{a}}$ & $9.25 \pm 0.48^{\mathrm{a}}$ & $12.25 \pm 0.95^{\mathrm{c}}$ & $13.00 \pm 0.71^{\mathrm{d}}$ & $12.00 \pm 0.00^{\mathrm{b}}$ & $10.50 \pm 0.29^{\mathrm{a}}$ & $10.75 \pm 0.48^{\mathrm{a}}$ & $10.75 \pm 0.25^{\mathrm{a}}$ & $11.25 \pm 0.25^{\mathrm{b}}$ & $11.00 \pm 0.91^{\mathrm{a}}$ & $10.25 \pm 0.25^{\mathrm{a}}$ \\
\hline & 40 & $13.25 \pm 0.63^{\mathrm{c}}$ & $6.75 \pm 0.25^{\mathrm{a}}$ & $11.25 \pm 0.75^{\mathrm{b}}$ & $11.25 \pm 0.25^{\mathrm{b}}$ & $14.00 \pm 0.71^{\mathrm{d}}$ & $17.00 \pm 1.23^{\mathrm{e}}$ & $9.75 \pm 0.48^{\mathrm{b}}$ & $13.75 \pm 0.48^{\mathrm{d}}$ & $10.00 \pm 0.00^{\mathrm{b}}$ & $12.75 \pm 1.11^{\mathrm{c}}$ & $9.75 \pm 0.48^{\mathrm{b}}$ \\
\hline & 30 & $11.75 \pm 0.75^{\mathrm{c}}$ & $6.25 \pm 0.25^{\mathrm{a}}$ & $10.50 \pm 0.65^{\mathrm{b}}$ & $20.00 \pm 1.78^{\mathrm{e}}$ & $10.75 \pm 0.75^{\mathrm{b}}$ & $16.00 \pm 1.35^{\mathrm{d}}$ & $9.50 \pm 0.29^{\mathrm{b}}$ & $10.50 \pm 0.29^{\mathrm{b}}$ & $9.25 \pm 0.25^{\mathrm{b}}$ & $9.75 \pm 0.25^{\mathrm{b}}$ & $8.75 \pm 0.48^{\mathrm{b}}$ \\
\hline & 20 & $13.75 \pm 0.75^{\mathrm{d}}$ & $10.75 \pm 0.48^{\mathrm{b}}$ & $9.75 \pm 0.48^{\mathrm{a}}$ & $13.75 \pm 0.63^{\mathrm{d}}$ & $12.25 \pm 0.75^{\mathrm{c}}$ & $14.25 \pm 1.44^{\mathrm{d}}$ & $8.50 \pm 0.29^{\mathrm{a}}$ & $8.00 \pm 0.00^{\mathrm{a}}$ & $8.75 \pm 0.48^{\mathrm{a}}$ & $9.50 \pm 0.65^{\mathrm{a}}$ & $13.00 \pm 0.91^{\mathrm{d}}$ \\
\hline & 10 & $10.25 \pm 0.25^{\mathrm{b}}$ & $9.00 \pm 0.00^{\mathrm{b}}$ & $9.00 \pm 0.00^{\mathrm{b}}$ & $15.75 \pm 2.72^{\mathrm{d}}$ & $13.25 \pm 1.03^{\mathrm{c}}$ & $11.25 \pm 0.95^{\mathrm{b}}$ & $10.00 \pm 0.00^{\mathrm{b}}$ & $0.00 \pm 0.00^{\mathrm{a}}$ & $10.25 \pm 0.25^{\mathrm{b}}$ & $11.25 \pm 0.63^{\mathrm{b}}$ & $18.75 \pm 1.49^{\mathrm{e}}$ \\
\hline \multirow{5}{*}{ S. enterica } & 50 & $10.00 \pm 0.00^{\mathrm{b}}$ & $0.00 \pm 0.00^{\mathrm{a}}$ & $11.75 \pm 0.25^{\mathrm{c}}$ & $18.75 \pm 0.75^{\mathrm{d}}$ & $12.00 \pm 0.41^{\mathrm{c}}$ & $11.75 \pm 1.11^{\mathrm{c}}$ & $18.25 \pm 1.18^{\mathrm{d}}$ & $17.25 \pm 1.32^{\mathrm{d}}$ & $11.75 \pm 0.25^{\mathrm{c}}$ & $11.50 \pm 0.96^{\mathrm{c}}$ & $9.00 \pm 0.71^{b}$ \\
\hline & 40 & $9.25 \pm 0.25^{\mathrm{b}}$ & $0.00 \pm 0.00^{\mathrm{a}}$ & $10.00 \pm 0.41^{\mathrm{b}}$ & $15.25 \pm 0.48^{\mathrm{e}}$ & $12.50 \pm 0.65^{\mathrm{d}}$ & $15.75 \pm 1.44^{\mathrm{e}}$ & $16.75 \pm 1.18^{\mathrm{e}}$ & $9.00 \pm 0.41^{\mathrm{b}}$ & $10.50 \pm 0.29^{\mathrm{c}}$ & $11.00 \pm 0.71^{\mathrm{c}}$ & $8.00 \pm 0.41^{\mathrm{b}}$ \\
\hline & 30 & $8.75 \pm 0.48^{\mathrm{a}}$ & $13.25 \pm 0.63^{\mathrm{a}}$ & $10.50 \pm 0.29^{\mathrm{a}}$ & $30.00 \pm 3.54^{\mathrm{d}}$ & $20.50 \pm 1.66^{\mathrm{c}}$ & $15.00 \pm 0.91^{\mathrm{b}}$ & $14.25 \pm 0.25^{\mathrm{b}}$ & $11.50 \pm 0.96^{\mathrm{a}}$ & $12.00 \pm 0.71^{\mathrm{a}}$ & $8.75 \pm 0.48^{\mathrm{a}}$ & $26.75 \pm 2.46^{d}$ \\
\hline & 20 & $7.50 \pm 0.29^{\mathrm{a}}$ & $9.25 \pm 0.25^{\mathrm{a}}$ & $13.25 \pm 0.63^{\mathrm{d}}$ & $12.50 \pm 0.65^{\mathrm{d}}$ & $14.00 \pm 0.91^{\mathrm{d}}$ & $10.00 \pm 0.00^{\mathrm{b}}$ & $14.25 \pm 0.25^{\mathrm{d}}$ & $10.00 \pm 0.00^{\mathrm{b}}$ & $18.25 \pm 1.18^{\mathrm{e}}$ & $12.25 \pm 1.25^{\mathrm{c}}$ & $23.00 \pm 1.35^{\mathrm{e}}$ \\
\hline & 10 & $8.75 \pm 0.25^{\mathrm{a}}$ & $8.75 \pm 0.48^{\mathrm{a}}$ & $9.25 \pm 0.25^{\mathrm{a}}$ & $9.75 \pm 0.25^{\mathrm{a}}$ & $26.00 \pm 1.00^{\mathrm{d}}$ & $9.50 \pm 0.50^{\mathrm{a}}$ & $12.75 \pm 0.75^{\mathrm{b}}$ & $8.75 \pm 0.25^{\mathrm{a}}$ & $18.75 \pm 1.25^{\mathrm{c}}$ & $10.25 \pm 0.25^{\mathrm{a}}$ & $18.75 \pm 0.75^{\mathrm{c}}$ \\
\hline \multirow{5}{*}{$\begin{array}{l}\text { K } \\
\text { pneumoniae }\end{array}$} & 50 & $11.25 \pm 0.48^{\mathrm{c}}$ & $9.75 \pm 0.25^{\mathrm{a}}$ & $12.50 \pm 0.87^{\mathrm{e}}$ & $13.75 \pm 0.48^{\mathrm{f}}$ & $12.00 \pm 1.00^{\mathrm{d}}$ & $16.75 \pm 1.18^{\mathrm{g}}$ & $13.00 \pm 0.41^{\mathrm{e}}$ & $9.00 \pm 0.58^{\mathrm{a}}$ & $10.00 \pm 0.00^{\mathrm{a}}$ & $11.00 \pm 0.71^{\mathrm{b}}$ & $8.50 \pm 0.29^{\mathrm{a}}$ \\
\hline & 40 & $18.25 \pm 1.18^{\mathrm{e}}$ & $8.75 \pm 0.48^{\mathrm{a}}$ & $12.50 \pm 1.04^{\mathrm{c}}$ & $11.75 \pm 0.25^{\mathrm{c}}$ & $10.50 \pm 0.29^{\mathrm{a}}$ & $15.00 \pm 1.47^{\mathrm{d}}$ & $11.00 \pm 0.41^{\mathrm{c}}$ & $9.00 \pm 0.00^{\mathrm{a}}$ & $9.00 \pm 0.00^{\mathrm{a}}$ & $16.00 \pm 1.41^{\mathrm{e}}$ & $8.25 \pm 0.48^{\mathrm{a}}$ \\
\hline & 30 & $6.75 \pm 0.48^{\mathrm{a}}$ & $9.00 \pm 0.71^{\mathrm{b}}$ & $10.25 \pm 0.25^{\mathrm{b}}$ & $17.00 \pm 1.47^{\mathrm{d}}$ & $10.00 \pm 0.00^{\mathrm{b}}$ & $15.25 \pm 0.85^{\mathrm{c}}$ & $10.50 \pm 0.50^{\mathrm{b}}$ & $10.50 \pm 0.29^{b}$ & $13.25 \pm 1.32^{\mathrm{c}}$ & $9.50 \pm 0.29^{\mathrm{b}}$ & $9.00 \pm 0.00^{\mathrm{b}}$ \\
\hline & 20 & $8.75 \pm 0.25^{\mathrm{a}}$ & $9.00 \pm 0.41^{\mathrm{a}}$ & $26.25 \pm 2.39^{\mathrm{d}}$ & $15.25 \pm 0.48^{\mathrm{c}}$ & $9.50 \pm 0.50^{\mathrm{a}}$ & $10.50 \pm 0.87^{\mathrm{a}}$ & $13.00 \pm 0.71^{\mathrm{b}}$ & $11.25 \pm 0.25^{\mathrm{a}}$ & $9.75 \pm 0.48^{\mathrm{a}}$ & $9.25 \pm 0.25^{\mathrm{a}}$ & $10.00 \pm 0.00^{\mathrm{a}}$ \\
\hline & 10 & $10.00 \pm 0.00^{\mathrm{b}}$ & $9.75 \pm 0.48^{\mathrm{b}}$ & $11.50 \pm 0.96^{\mathrm{b}}$ & $17.25 \pm 1.32^{\mathrm{c}}$ & $7.75 \pm 0.25^{\mathrm{a}}$ & $10.75 \pm 0.85^{\mathrm{b}}$ & $15.50 \pm 0.87^{\mathrm{c}}$ & $10.25 \pm 0.25^{\mathrm{b}}$ & $10.50 \pm 0.50^{\mathrm{b}}$ & $11.25 \pm 0.63^{\mathrm{b}}$ & $7.50 \pm 0.29^{\mathrm{a}}$ \\
\hline \multirow{5}{*}{ S. aureus } & 50 & $12.00 \pm 0.58^{\mathrm{a}}$ & $10.75 \pm 0.25^{\mathrm{a}}$ & $11.25 \pm 0.48^{\mathrm{a}}$ & $13.75 \pm 0.48^{b}$ & $21.25 \pm 2.39^{\mathrm{c}}$ & $19.50 \pm 0.87^{c}$ & $30.00 \pm 0.00^{\mathrm{d}}$ & $13.00 \pm 0.71^{\mathrm{a}}$ & $11.75 \pm 0.48^{\mathrm{a}}$ & $11.00 \pm 0.41^{\mathrm{a}}$ & $11.25 \pm 0.48^{\mathrm{a}}$ \\
\hline & 40 & $14.50 \pm 0.50^{\mathrm{b}}$ & $9.50 \pm 0.29^{\mathrm{a}}$ & $23.50 \pm 0.96^{\mathrm{f}}$ & $11.25 \pm 0.25^{\mathrm{a}}$ & $13.75 \pm 0.48^{b}$ & $17.75 \pm 1.25^{\mathrm{c}}$ & $12.00 \pm 0.00^{\mathrm{a}}$ & $11.25 \pm 0.48^{\mathrm{a}}$ & $18.50 \pm 1.26^{\mathrm{d}}$ & $21.50 \pm 3.01^{\mathrm{e}}$ & $9.75 \pm 0.25^{\mathrm{a}}$ \\
\hline & 30 & $13.75 \pm 1.03^{\mathrm{b}}$ & $9.75 \pm 0.25^{\mathrm{a}}$ & $20.00 \pm 1.23^{\mathrm{e}}$ & $13.75 \pm 0.48^{\mathrm{b}}$ & $15.75 \pm 3.09^{\mathrm{c}}$ & $17.50 \pm 1.66^{\mathrm{d}}$ & $14.25 \pm 0.25^{\mathrm{b}}$ & $9.75 \pm 0.48^{\mathrm{a}}$ & $12.25 \pm 0.48^{\mathrm{a}}$ & $11.25 \pm 0.63^{\mathrm{a}}$ & $14.25 \pm 0.48^{b}$ \\
\hline & 20 & $10.50 \pm 0.29^{\mathrm{a}}$ & $10.00 \pm 0.41^{\mathrm{a}}$ & $20.75 \pm 1.70^{\mathrm{d}}$ & $16.25 \pm 1.25^{\mathrm{c}}$ & $13.25 \pm 0.25^{\mathrm{b}}$ & $16.75 \pm 1.44^{\mathrm{c}}$ & $15.75 \pm 0.48^{\mathrm{c}}$ & $11.75 \pm 0.75^{\mathrm{a}}$ & $9.50 \pm 0.50^{\mathrm{a}}$ & $11.25 \pm 0.25^{\mathrm{a}}$ & $10.75 \pm 0.25^{\mathrm{a}}$ \\
\hline & 10 & $8.00 \pm 0.00^{\mathrm{a}}$ & $8.25 \pm 0.25^{\mathrm{a}}$ & $13.75 \pm 0.48^{\mathrm{b}}$ & $17.25 \pm 1.03^{\mathrm{c}}$ & $19.25 \pm 0.75^{\mathrm{c}}$ & $13.00 \pm 1.16^{\mathrm{b}}$ & $20.75 \pm 1.11^{\mathrm{d}}$ & $13.25 \pm 1.11^{\mathrm{b}}$ & $8.00 \pm 0.41^{\mathrm{a}}$ & $10.25 \pm 0.48^{\mathrm{a}}$ & $21.75 \pm 1.18^{\mathrm{e}}$ \\
\hline
\end{tabular}

Key: B: Bryophyllum pinnatum; O: Ocimum gratissimum; J: Jathropha curcas; F: Ficus experata; a-f: different characters in the same row indicate values with significant difference (p $<0.05$ ) 
Conclusion: Phytochemicals present in the leave extracts of B. pinnatum, O. graticcimum, J. curcas and $F$. exasperate enhanced their antibacterial activities in combinations. S. aureus and E. coli had the highest zones of inhibition observed in the study with a combination of Ocimum gratissimum and Ficus exasperate for $S$. aureus, and Bryophyllum pinnatum and Ocimum gratissimum for E. coli. Studies on combined plant extracts interactions enhance their bioavailability and antibacterial activity as compared to studies on single extracts. Therefore, they can be good sources of antibacterial drugs.

\section{REFERENCES}

Abbas, AA; Ernest, BA; Akeh, M; Upla, P; Tuluma, TK (2017). Antimicrobial activity of coconut oil and its derivative (lauric acid) on some selected clinical isolates. Int. J. Med. Sci. Clin. Invent. 4(8):3173 - 3177.

Aggarwal, B; Lamba, HS; Ajeet, PS (2017). Various pharmacological aspects of Cocos nucifera - A review. Am. J. Pharmacol. Sci. 5(2):25 - 30.

Akinjogunla, OJ; Adegoke, AA; Udokang, IP; Adebayo-Tayo, BC (2009). Antimicrobial potential of Nymphoea lotus (Nymophaeceae) against wound pathogens. J. Med. Plant. Res. 3(3):138 - 141.

Awala, SI; Ajayi, OE; Alabi, OA; Ajayi, O; Olalekan, OT (2017). Exploration of the antimicrobial properties of Ficus exasperata leaves from Akure metropolis. Adv. Res. 9: 1 - 6

Chouhan, S; Sharma, K; Guleria, S (2017). Antimicrobial activity of some essential oilspresent status and future perspectives. Medicine 4(58): 1 - 21.

Costa, DC; Costa, HS; Albuquerque, TG; Ramos, F; Castilho, MC; Sanches-Silva, A (2015). Advances in phenolic compounds analysis of aromatic plants and their potential applications. Trends Food. Sci. Technol. 45:336 - 354.

D’Arrigo, M., Ginestra, G., Mandalari, G., Furneri, P.M. and Bisignano, G. (2010). Synergism and post antibiotic effect of tobramycin and Melaleuca alternifolia (tea tree) oil against Staphylococcus aureus and Escherichia coli. Phytomedicine 17:317 - 322.

Dawoud, MEA; Mawgoud, YA; Gouda-Dawoud, TM (2013). Synergistic interactions between plant extracts, some antibiotics and/or their impact upon antibiotic-resistant bacterial isolates. Afr. $J$. Biotechnol. 12(24):3835 - 3846.
Harborne, J (1973). Phytochemical methods: A guide to modern techniques of plant analysis. 2nd Edition, Chapman and Hall Ltd, London

Ildiz, N; Baldemir, A; Ince, U; Ilgun, S; Konca, Y (2018). Synergistic effect of Coriander sativum L. extracts with cefoxitin against methicillin resistant Staphylococcus aureus, extendedspectrum beta-lactamase producing Escherichia coli and Klebsiella pneumoniae. Med. Sci. 4:777 $-780$

Karmegam, N; Karuppusamy, S; Prakash, MK; Jayakumar, M. Rajasekar, K (2008). Antibacterial potency and synergistic effect of certain plant extracts against food-borne diarrheagenic bacteria. Int. J. Biomed. Pharmaceut. Sci. 2:88 - 93.

Kin, A., Yaki, L. M., Abubakar, I., Olusola, L. F., Zubairu, R. (2018). Antibacterial activity of Ocimum gratissimum (scent leaf) on some pathogenic gastrointestinal bacteria. Afr. J. Microbiol. Res. 12: 923 - 929

Ladipo, MK; Doherty, VF; Kanife, UC (2010). Phytochemical screening and antibacterial investigation of the extract of Ocimum gratissimum (Scent Leaf) on selected enterobacteriaceae. Production, Agriculture and Technology (PAT) 6(2):75 - 84.

Obadoni, B; Ochuko, P (2001). Phytochemical studies and comparative efficacy of the crude extracts of some homostatic plants in Edo and Delta States of Nigeria. Global J. Pure Appl. Sci. 8:203-- 208

Odey, MO; Iwara, IA; Udiba, UU; Johnson, JT; Inekwe, UV; Asenye, ME; Victor, O (2012). Preparation of plant extracts from indigenous medicinal plants. Int. J. Sci. Technol. 1(12):688 - 692 .

Ogbeibu, AE (2014). Biostatistics: A Practical Approach to Research and Data Handling. 2nd edition, Mindex, Benin.

Prakash, M; Karthikeyan, V; Karuppusamy, S; Karmegam, N (2006). Synergistic activity of certain plants extracts against methicillin resistant Staphylococcus aureus (MRSA). J. Ecotox. Env. Monit.16:387 - 390.

Rakholiya, K; Chanda, S (2012). In vitro interaction of certain antimicrobial agents in combination with plant extracts against some pathogenic bacterial strains. Asian. Pac. J. Trop. Biomed. 2:876 - 880.

Rojas, R; Bustamante, B; Bauer I (2004). Antimicrobial activity of selected Peruvian 
medicinal plants. J. Ethnopharmacol. 88: 199 204.

Rukmini, JN; Manasa, S; Rohini, C; Sireesha, LP; Ritu, S; Umashankar, GK (2017). Antibacterial efficacy of tender coconut water (Cocos nucifera L) on Streptococcus mutans: An in-vitro study. J. Int. Soc.Prevent. Communit. Dent. 7:130 - 134.

Stefanović, O; Comic, L (2012). Synergistic antibacterial interaction between Melissa officinalis extracts and antibiotics. J.Appl. Pharm. Sci. 2(1):1 - 5.

Terezinha, JF; Rafael, SF; Lidiane, y; Jose, R; Pinto, S; Noemia, KI; Aneli, MB (2008). Antifungal activity of essential oil isolated from Occimum gratissimum L. (eugenoichemotype) against phytopathogenic fungi. Braz. Arch. Biol. Technol. 49(6): 867 - 891.
Tyagi, N; Hooda, V; Hooda, A; Malkani, S (2017). Evaluation of antidiabetic potential of ethanolic and aqueous extract of Cocos nucifera endocarp. World J. Pharm. Pharma. Sci. 4(7):1112 - 1120.

Udochukwu, U; Omeje, FI; Uloma, IS; Oseiwe, FD (2015). Phytochemical analysis of Vernonia amygdalina and Ocimum gratissimum extracts and their antibacterial activity on some drug resistant bacteria. Am. J. Res. Commun. 3(5):225 -235 .

WHO, (2000). Expert Committee on medicinal importance of native plants. Technical report series. WHO, Geneva.

Złotek, U; Mikulska, S; Nagajek, M; S'wieca, M (2016). The effect of different solvents and number of extraction steps on the polyphenol content and antioxidant capacity of basil leaves (Ocimum basilicum L.) extracts. Saudi. J. of Biol. Sci. 23:628 - 633. 